Research Article

\title{
Defects-Induced Hot Spots in TATB
}

\author{
Zhonghua Yan, ${ }^{1,2}$ Chuanchao Zhang, ${ }^{1}$ Hongwei Yan, ${ }^{1}$ Zhijie Li, ${ }^{2}$ \\ Li Li, ${ }^{2}$ Ming Huang, ${ }^{3}$ Bisheng Tan, ${ }^{3}$ and Xiaodong Yuan ${ }^{1}$ \\ ${ }^{1}$ Research Center of Laser Fusion, China Academy of Engineering Physics, Mianyang 621900, China \\ ${ }^{2}$ School of Physical Electronics, University of Electronic Science and Technology of China, Chengdu 610054, China \\ ${ }^{3}$ Institute of Chemical Materials, China Academy of Engineering Physics, Mianyang 621900, China
}

Correspondence should be addressed to Hongwei Yan; hwyan@163.com and Xiaodong Yuan; yxd66my@163.com

Received 1 March 2014; Accepted 23 May 2014; Published 17 June 2014

Academic Editor: Kai Sun

Copyright (C) 2014 Zhonghua Yan et al. This is an open access article distributed under the Creative Commons Attribution License, which permits unrestricted use, distribution, and reproduction in any medium, provided the original work is properly cited.

\begin{abstract}
We investigate the interaction between the laser and energetic materials with different defects. The three-dimensional models of triaminotrinitrobenzene (TATB) explosives containing spherical pores, craters, and cracks are established, respectively. The laser ignition process of TATB is simulated with three-dimensional finite difference time domain (3D-FDTD) method to study the electromagnetic field distribution surrounding these defects with $355 \mathrm{~nm}$ laser incidence. It indicates that the larger defects in the TATB energetic materials have the stronger electric field modulations to initial incident laser for all the three defects, which is easier to lead to the generation of hot spots. Furthermore, TATB materials with spherical pore defects and crater defects are easier to form hot spots than those with narrow crack defects.
\end{abstract}

\section{Introduction}

The ignition of explosives has been studied for so many years. There are several conventional means for the ignition systems, such as electric spark ignition and electric bridge-wire ignition. However, due to the interference of static electricity, radio frequency, electromagnetic field, and other unpredictable factors, traditional ignition technologies of explosives materials tend to explode by mistake or fail to work.

Laser ignition technology, introduced by Brish et al. [1] and Yang and Menichelli [2] in the late sixties and early seventies of last century, has been recognized as a safe and reliable means which has extensive potential applications in the aerospace and military filed owing to the laser's high intensity and good direction. The first discussion of laser ignition mechanisms is also discussed in [1]. Generally speaking, there are two possible ignition mechanisms used to interpret the explosion phenomenon: thermal ignition mechanism and impulse ignition mechanism. In the thermal mechanism, the intense local heating by the laser pulse causes the rate of chemical reactions within the materials to accelerate sharply. It requires flux densities in the neighborhood of $1 \mathrm{~kW} / \mathrm{cm}^{2}$ or less and pulse durations of about $1 \mathrm{~ms}$ or longer. But in the impulse ignition mechanism, where the chemical reactions are accelerated by a strong laser-generated shock wave, it needs more intense and shorter duration by factors of about $10^{6}$ with the similar energy content than that in the thermal ignition mechanism [3]. The term "hot spots" represents the regions which can couple efficiently the locally higher temperature and ultimately initiation in materials [4]. Because of their modulations for the laser electromagnetic field, defects (voids, bubbles, etc.) in the explosives materials can result in the generation of hot spots.

Based on the thermal ignition mechanism, we investigated the interaction between the laser and energetic materials with different defects in our work. Triaminotrinitrobenzene (TATB) explosives were firstly characterized with the Scanning Electron Microscopy (SEM). Three-dimensional (3D) models of TATB energetic materials containing spherical pore, crater, and crack defects were established, respectively. The laser ignition process of TATB was simulated with three-dimensional finite difference time domain (3D-FDTD) 


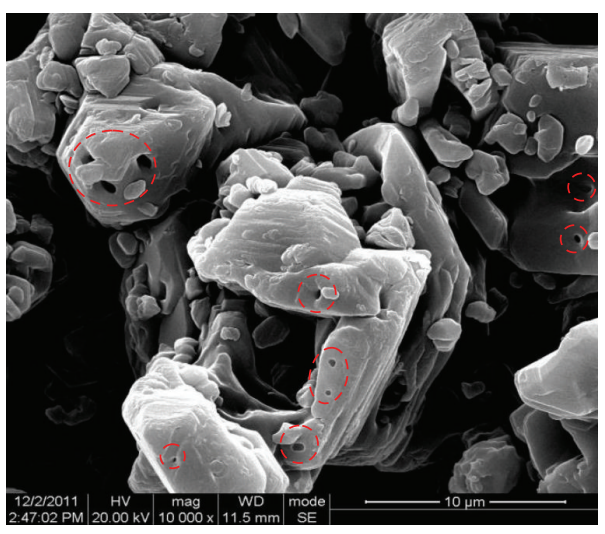

(a)

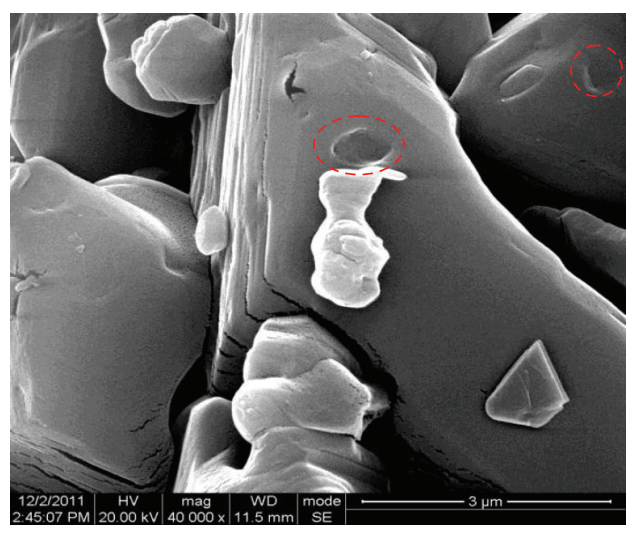

(b)

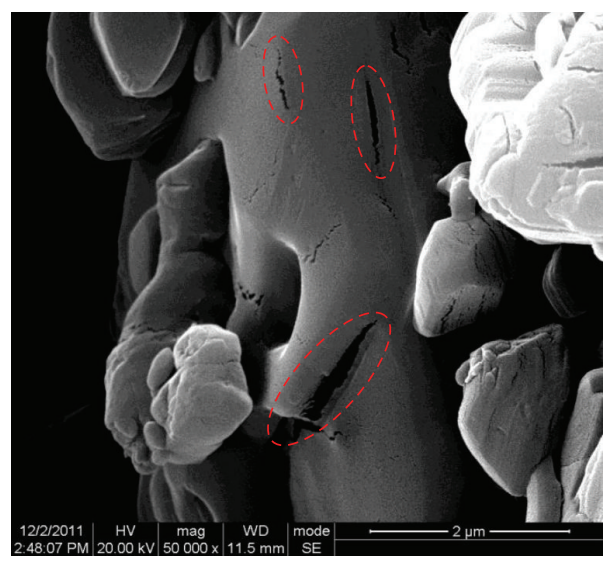

(c)

FIGURE 1: SEM of TATB explosives with different defects: (a) spherical pores, (b) craters, and (c) cracks.

TABLE 1: The size range of different defects.

\begin{tabular}{|c|c|c|}
\hline \multirow{2}{*}{ Defects } & \multicolumn{2}{|c|}{ Size range } \\
\hline & SEM & FDTD simulations \\
\hline Spherical pores & $r=0.5-1.5 \mu \mathrm{m}$ & $r=20-60 \delta$ \\
\hline Craters $(a \geq b)$ & $a=0.5-3 \mu \mathrm{m}$ & $a=20-100 \delta$ \\
\hline $\operatorname{Cracks}(d \geq w)$ & $l=0.5-3 \mu \mathrm{m}$ & $l=20-100 \delta, w=0.2 * l$ \\
\hline
\end{tabular}

method [5-8] to study the electromagnetic field distribution (in proportion to the flux density I) surrounding these defects with $355 \mathrm{~nm}$ laser incidence. The simulated results of electric field amplitude distributions and the number of highintensity spots in TATB materials were analyzed to investigate the effect of the three kinds of defects on the generation of hot spots.

\section{Models and FDTD Method}

The TATB explosives with many microdefects, including spherical pores, craters, and cracks, were characterized with the Scanning Electron Microscopy (SEM) shown in Figure 1.
It indicated that all the defect sizes are in the micron or submicron level (shown in Table 1). As it is difficult to know the light modulation of only one of these defects through experiments, in this work we utilized the 3D-FDTD methods to simulate the modulation of each defect to the incident laser. According to these SEM images, we constructed the threedimensional sphere model, rotated parabolic body model, and straight triangular prism model, respectively, to fit the air area as encircled in Figure 1, as their three-dimensional profiles showed in Figure 2. We supposed that all the defects lie in the top surface of the TATB explosives. Their equations in Cartesian coordinates are as follows.

Sphere

$$
\left(z-z_{0}+h-r\right)^{2} \leq r^{2}-\left[\left(x-x_{0}\right)^{2}+\left(y-y_{0}\right)^{2}\right] .
$$

Rotated parabolic body

$$
z \geq \frac{4 b}{a^{2}}\left[\left(x-x_{0}\right)^{2}+\left(y-y_{0}\right)^{2}\right]+z_{0}-b .
$$




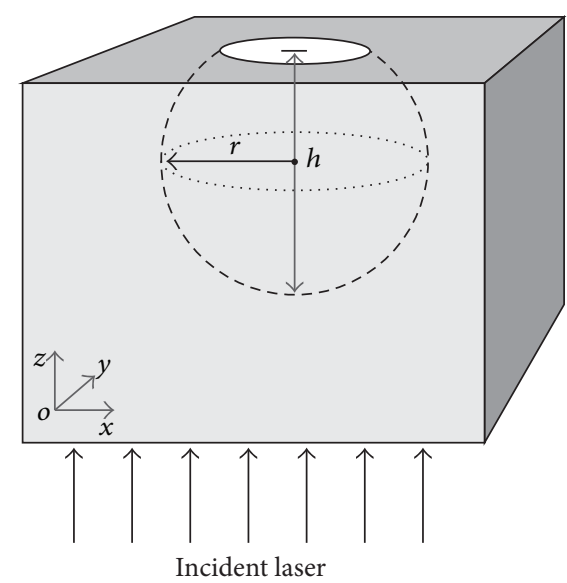

(a)

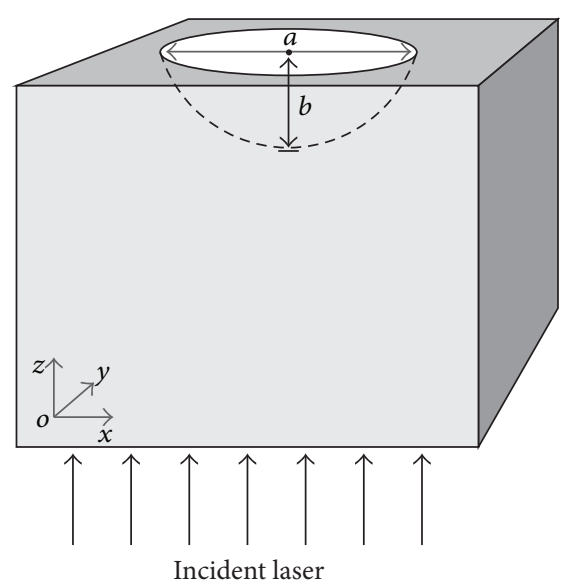

(b)

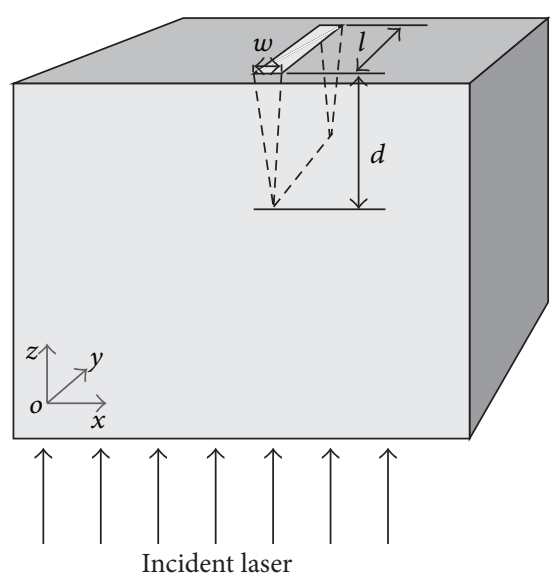

(c)

FIGURE 2: The three-dimensional profiles of models for the microdefects in Figure 1: (a) sphere, (b) rotated parabolic body, and (c) straight triangular prism.

Straight triangular prism

$$
\begin{gathered}
z \geq \frac{2 d}{w}\left(x-x_{0}\right)+z_{0}-d, \\
z \geq-\frac{2 d}{w}\left(x-x_{0}\right)+z_{0}-d, \\
y_{0}-\frac{l}{2} \leq y \leq y_{0}+\frac{l}{2},
\end{gathered}
$$

where $\left(x_{0}, y_{0}, z_{0}\right)$ is the coordinate of the symmetrical center of the top surface. $r$ and $h$ represent the radius and the depth of the spherical pores, respectively. $a$ and $b$ are the width and depth of the craters. $l, d$ and $w$ represent the length, the depth and the width of the cracks respectively. From the SEM, we assumed that $a \geq b, d \geq w$. For easy description, in our study we supposed that the depth/radius ratio of spherical pore defects Rs $=h / r$, the depth/width ratio of crater defects $\mathrm{Rcr}=$ $b / a$, and the depth/width ratio of crack defects $\mathrm{Rck}=d / w$.

The finite difference time domain (FDTD) method, proposed by Yee in 1966 [9], has been proven to be one of the most popular and powerful numerical tools for simulating the propagation and scattering of classical electromagnetic fields in materials. It is a solution method of the partial derivatives in Maxwell's equations by discretizing these equations in time and space domains using the difference scheme on edges of the "Yee-cell" [9], which need to divide space and time into a regular grid. The field solution at the current time step is deduced from the field values at the previous time steps with the recursive time-marching algorithm.

In our study, the overall lattice in the TATB explosives is a uniformly gridded cuboid simulation domain as a dimension of $200 \times 200 \times 215$ cells with the same unit cell size for the three directions $\Delta x=\Delta y=\Delta z=\delta=\lambda / 12$ and a time step $\Delta t=\delta / 2 v$ to satisfy computational stability and numerical dispersion conditions, where $\lambda$ is the $355 \mathrm{~nm}$ laser wavelength and $v$ is the velocity of light in the air. The time step number $n$ is 1600 for all three models. The normal incidence laser was from the bottom of the model with the electric field amplitude $(|E|) 1.0 \mathrm{~V} / \mathrm{m}$ shown in Figure 2. The relative dielectric constants $\left(\varepsilon_{r}\right)$ of air and TATB are 1.0 and 4.0 , respectively [10]. The boundaries are terminated by a $9 \delta$ thickness perfectly matched layer (PML) [11].

\section{Results and Discussion}

Equation (4) expounds the relationship of the flux density $I$ and electric field amplitude $|E|$. As we know, the flux density distribution can be analyzed by the electric field amplitude in the TATB explosives

$$
I \propto|E|^{2} .
$$

3.1. Spherical Pore Defects. In order to explore effects of the radius $r$ of spherical pore defects and their depth $h$ embedded in TATB explosives on the flux density distribution, we investigated the relationship between the electric field distribution and the depth/radius ratio Rs. The size range of FDTD simulations is shown in Table 1. Figure 3 shows the maximum electric field $|E|_{\max }$ of some $z$ planes at different Rs when $r=60 \delta$. As the location of $z$ plane changes, $|E|_{\max }$ fluctuates up and down at different $z$ planes. However, the general trend increases gradually. Figure 4(a) shows the maximum electric field $|E|_{\text {max }}$ of Plane $x o z(y=y 0)$ at the radius $r=40 \delta$ and the whole simulation domain at $r=20 \delta, 40 \delta$, and $60 \delta$ changing with the increasing Rs. It is obvious that $|E|_{\max }$ of all the simulation domain is not obtained definitely at Plane xoz. It is even much larger than that in the Plane xoz. As Rs increases from 0.125 to $2.5,|E|_{\max }$ for the whole space changes in the range of $2.0-2.7 \mathrm{~V} / \mathrm{m}$, and the flux density reaches a factor (light intensity enhancement factor, LIEF) of 4-7.29 compared with the initial incident laser. It is clear that the larger the radius of sphere defects is, the stronger its modulation to incident laser is. When the size of spherical pores is small, the modulation effect is weak because of the minor scattering 


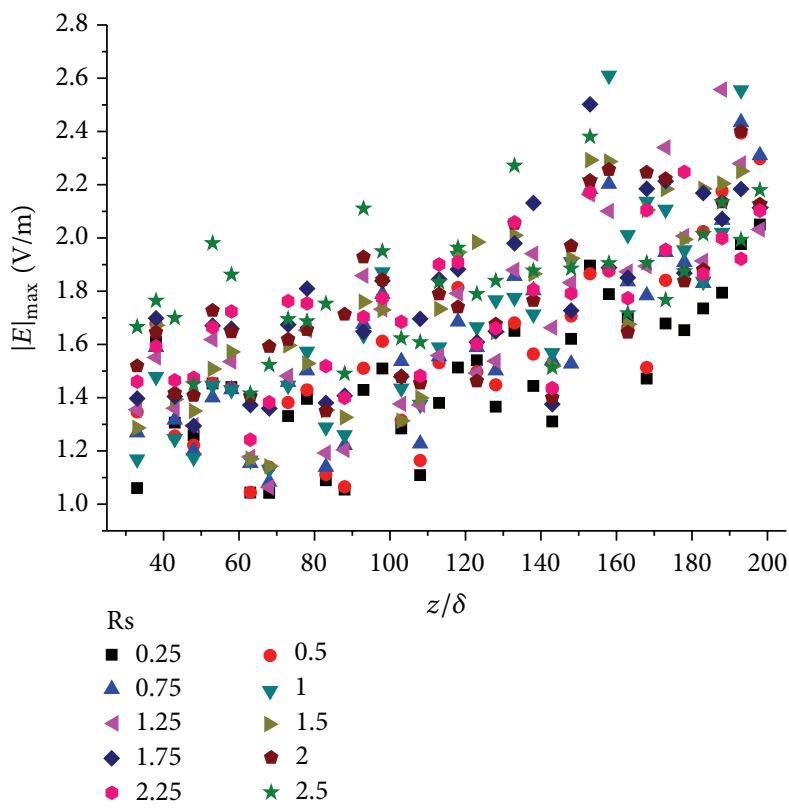

FIGURE 3: $|E|_{\max }$ distributions at different $z$ planes with various Rs at $r=60 \delta$.

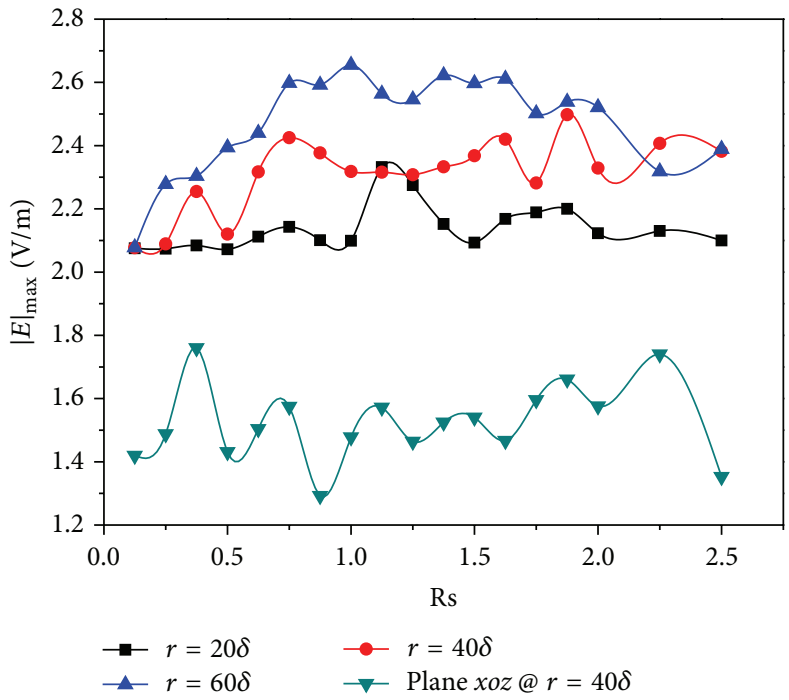

(a)

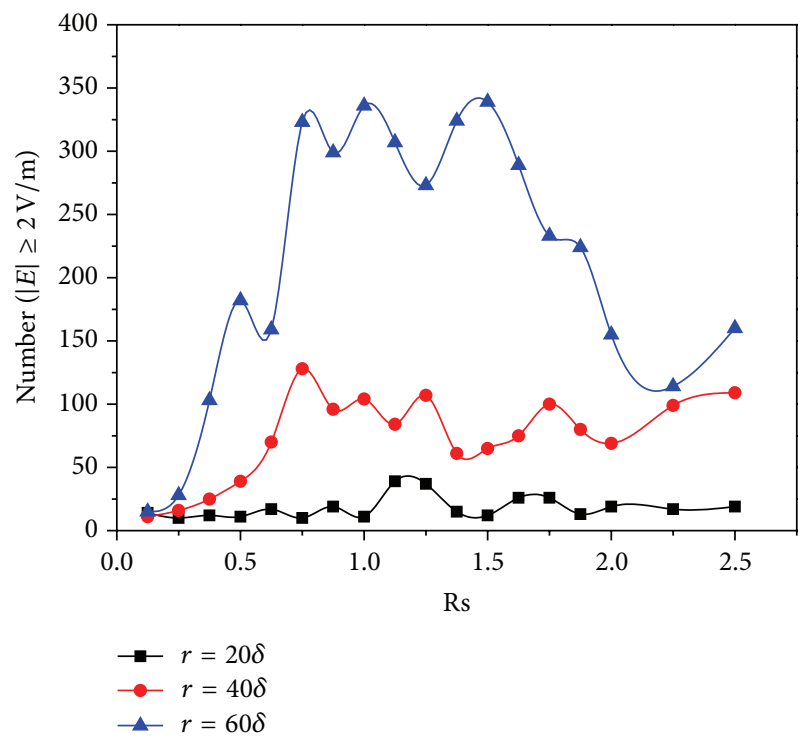

(b)

FIGURE 4: (a) $|E|_{\max }$ and (b) the number of high-intensity spots at various Rs.

surface. However, when $r$ gets to $60 \delta$, the reflecting surface increases owing to the increasing surface area. At this time, even total reflection may appear. So the modulation to initial laser is stronger when sphere is larger.

Besides, we use the term "high-intensity spot" in this work to express the region where the electric field amplitude $|E| \geq 2.0 \mathrm{~V} / \mathrm{m}$ and the number of high-intensity spots was counted and exported by computer program. Figure 4(b) shows the number of high-intensity spots in the whole simulation space. It revealed that the number increases firstly with the increase of Rs and then decreases to a stable range when Rs $\geq 2$, in which the defects have been completely embedded into the explosives. As the radius $r$ increases, the number of high-intensity spots increases. It shows that the number of high-intensity spots induced by spherical pore defects when $r=1.8 \mu \mathrm{m}(60 \delta)$ has reached about 3 times of that when $r=1.2 \mu \mathrm{m}(40 \delta)$.

3.2. Crater Defects. The interaction between laser and TATB explosives containing crater defects is also studied. 


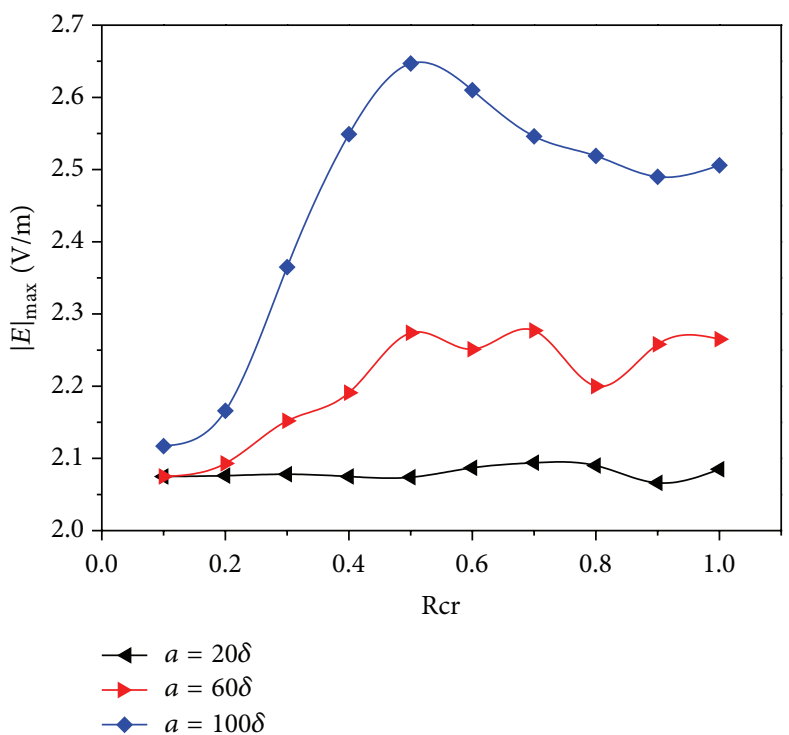

(a)

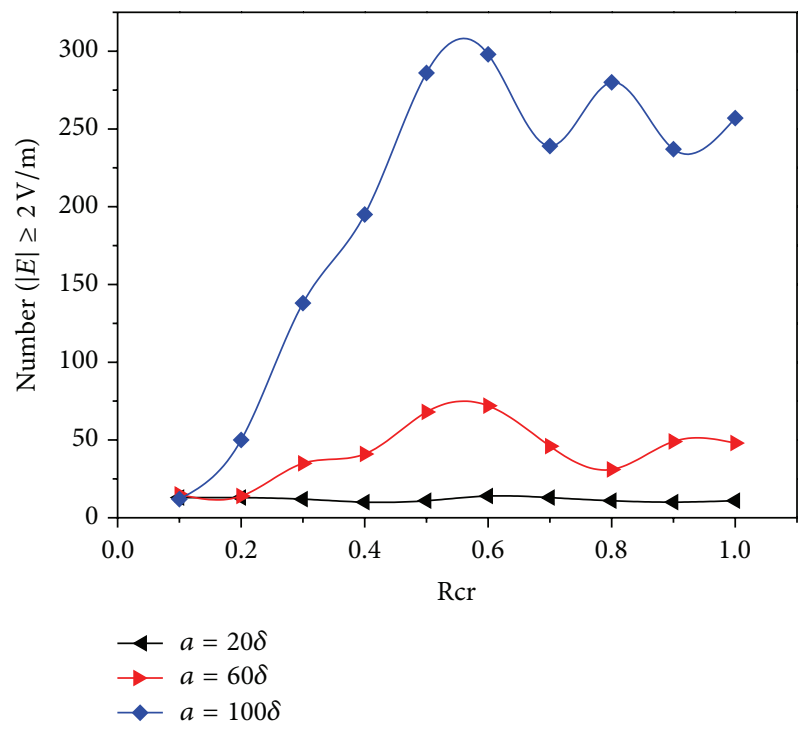

(b)

Figure 5: (a) $|E|_{\max }$ and (b) the number of high-intensity spots as the function of Rcr.

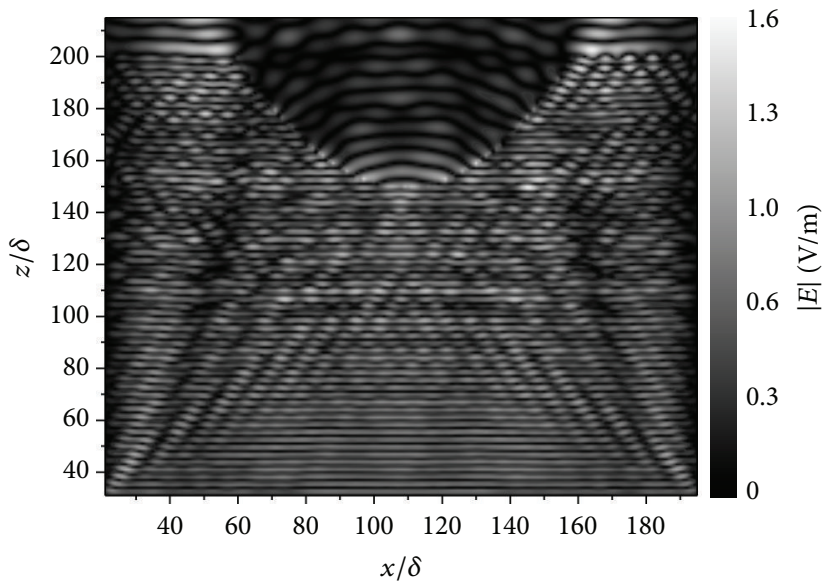

(a)

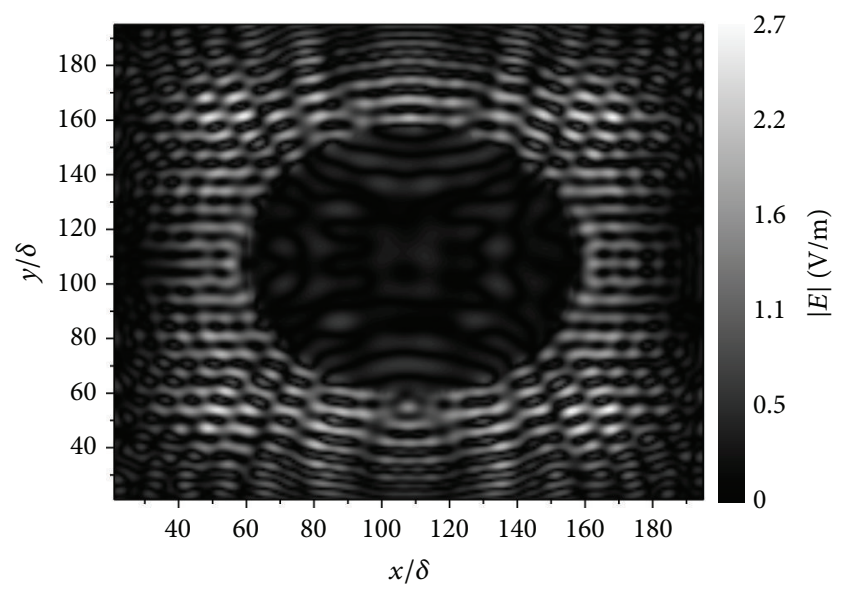

(b)

FIGURE 6: Electric field amplitude distributions when $a=100 \delta$ and $\mathrm{Rcr}=0.5$ : (a) Plane $x o z$ and (b) Plane $z 198$ where the maximum electric field amplitude occurs.

Figure 5 shows the effects of the width $a$ and depth $b$ of crater defects (shown in Figure 2(b)) on the electric field distributions. Generally speaking, the maximum electric field $|E|_{\max }$ increases firstly with the increasing depth/width ratio Rcr and then decreases slowly. The larger the width of crater defects is, the stronger its modulation to incident laser is. When $a=0.6 \mu \mathrm{m}(20 \delta),|E|_{\max }$ keeps basically in a stable value around $2.08 \mathrm{~V} / \mathrm{m}$ and there is a small number of highintensity spots. However, when $a$ gets to $1.8 \mu \mathrm{m}(60 \delta),|E|_{\max }$ is mainly in the range of $2.1-2.3 \mathrm{~V} / \mathrm{m}$ with the LIEF from 4.41 to 5.29 as Rcr changes. The number of high-intensity spots increases to dozens. Finally, when $a$ reaches up to $3 \mu \mathrm{m}$ $(100 \delta)$, most of the maximum electric field $|E|_{\max }$ keep in the level above $2.55 \mathrm{~V} / \mathrm{m}$ with the LIEF more than 6.5 times compared to the initial incident laser. Similar to the spherical pore defects, as the width of crater defects $a$ increases, the number of high-intensity spots increases. It shows that the number of high-intensity spots when $a=3 \mu \mathrm{m}$ has reached about 4 times of that when $a=1.8 \mu \mathrm{m}$.

Figure 6, respectively, displays the electric field amplitude distributions of Plane $x o z(y=y 0)$ and Plane $z 198$ (where the maximum electric field amplitude occurs) when $a=100 \delta$, $\mathrm{Rcr}=0.5$. As the defects lie in the top surface, the electric field near the top surface is higher. And the maximum electric field appears in Plane $z 198$ which is nearly close to crater defects.

3.3. Crack Defects. According to Figure 2(c), we investigated the effect of the depth/width ratio Rck of crack defects with 


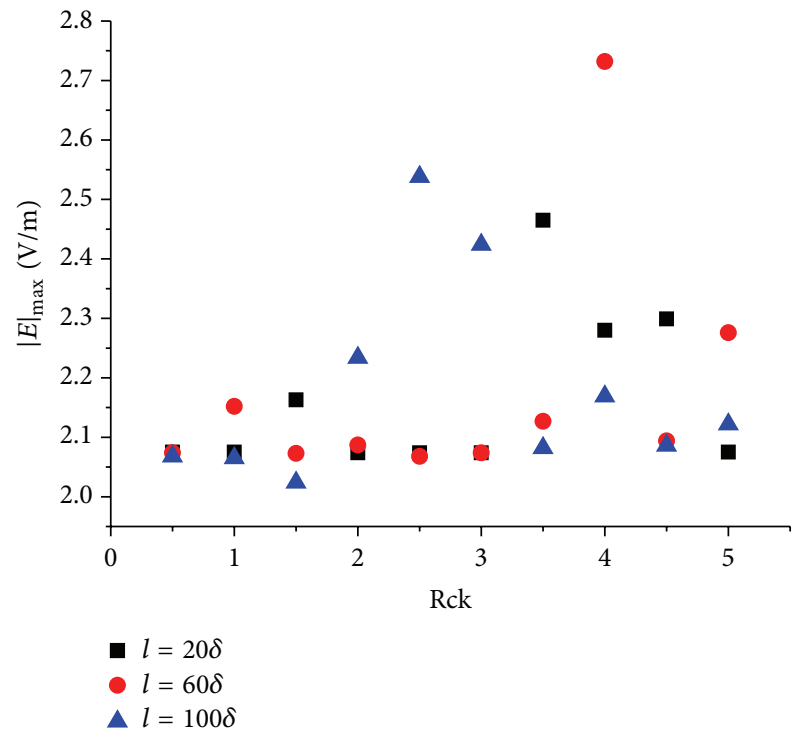

(a)

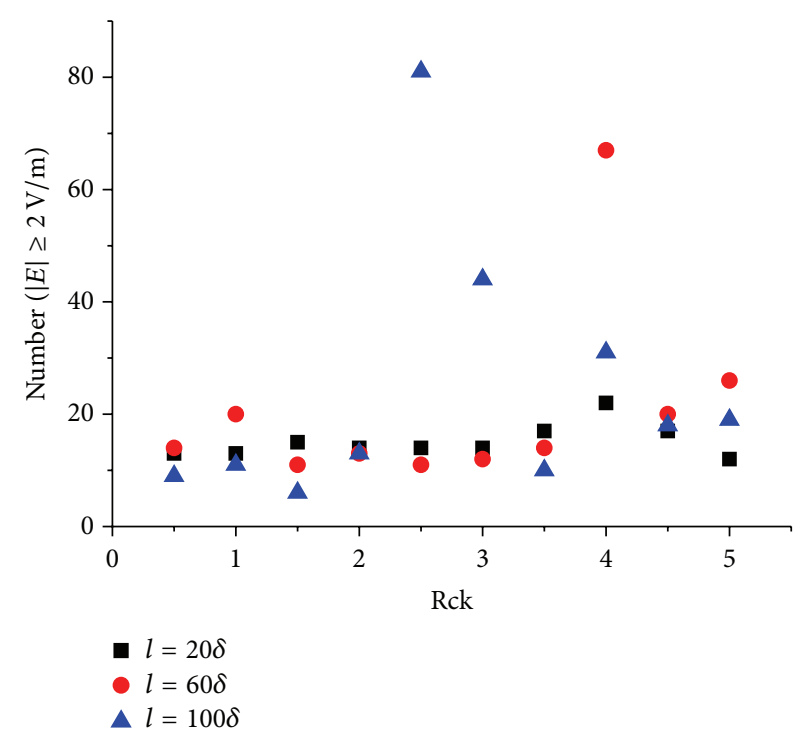

(b)

Figure 7: (a) $|E|_{\max }$ and (b) the number of high-intensity spots as the function of Rck.

various length $l=20 \delta, 60 \delta$, and $100 \delta$ on the propagation and scattering of electromagnetic fields within the TATB explosives. From the SEM image in Figure 1(c), we assumed the width $w=l / 5$. Figure 7(a) displays the maximum electric field amplitude $|E|_{\max }$ changing with the increasing Rck. As the length of crack defects changes, most $|E|_{\max }$ lie in the range of $2.1-2.3 \mathrm{~V} / \mathrm{m}$ with the number of high-intensity spots less than 30 . As the length of crack defects increases, there are also some enhanced electric field areas. However, it is not so many as those of crater and crack defects because of the narrow width.

The Rck for gaining the maximum of $|E|_{\max }$ is different for different lengths. When $l$ is about $0.6 \mu \mathrm{m}(20 \delta)$, the maximum, $2.47 \mathrm{~V} / \mathrm{m}$, is obtained at Rck $=3.5$. However, for $l=1.8 \mu \mathrm{m}(60 \delta)$ and $3 \mu \mathrm{m}(100 \delta)$, Rck changes to 4.5 and 2.5 with their $|E|_{\max }$ values to $2.73 \mathrm{~V} / \mathrm{m}$ and $2.54 \mathrm{~V} / \mathrm{m}$, respectively. Even so, the maximal number of high intensity spots is acquired at $l=100 \delta$ when Rck $=2.5$. Figure 8 shows the electric field amplitude distributions in the $z$ plane of $|E|_{\max }$ at the length $l=100 \delta$ and width $w=20 \delta$. It indicates that every $|E|_{\max }$ lies in the $z$ plane which is on (Plane $z 200$ ) or near (Plane $z 187, z 193$ ) the top surface. Especially, the $|E|_{\max }$ lie in the $z$ plane of the bottom of crack defects (Plane $z 141$ for Rck $=3$, Plane $z 121$ for Rck $=4$ ).

From Figures 4, 5, and 7, we can deduce that the larger the defect in the TATB energetic material is, the stronger modulation to incident laser is, which is easier to generate hot spots. Moreover, spherical pore defects when $r=1.8 \mu \mathrm{m}$ with Rs from 0.75 to 1.75 and crater defects when $a=3 \mu \mathrm{m}$ with Rcr from 0.4 to 1.0 are easier to lead to the generation of hot spots than crack defects with $w=l / 5$ because of the narrow width.

\section{Conclusions}

The laser interactions with TATB explosives including different defects (spherical pores, craters, and cracks) were investigated, respectively, with the three-dimensional finite difference time domain method. The modulations of these defects to incident laser were analyzed with the maximum electric field amplitude and the number of high-intensity spots.

The results indicate that the maximum electric field $|E|_{\max }$ of spherical pores changes in the range of $2.0-2.7 \mathrm{~V} / \mathrm{m}$ as the radius $r$ changes from 0.6 to $1.8 \mu \mathrm{m}$ with the depth/radius ratio Rs increasing from 0.125 to 2.5 . And the light intensity enhancement factor (LIEF) reaches 4-7.29 compared with the initial laser. As the radius $r$ increases, electric field amplitude strengthens and the number of high-intensity spots increases. The number of high-intensity spots induced by spherical pore defects when $r=1.8 \mu \mathrm{m}$ reaches about 3 times of that when $r=1.2 \mu \mathrm{m}$. Similar to the spherical pore defects, as the width $a$ of crater defects increases from 0.6 to $3 \mu \mathrm{m}$, the number of high-intensity spots increases to several hundred. When $a=3 \mu \mathrm{m}$, the number of high-intensity spots has reached about 4 times of that when $a=1.8 \mu \mathrm{m}$ with most of the maximum electric field in the level above $2.55 \mathrm{~V} / \mathrm{m}$ (LIEF $\geq$ 6.5). However, as the length of crack defects $l$ changes from 0.6 to $3 \mu \mathrm{m}$ with the width $w=l / 5$, most $|E|_{\max }$ lie in the range of $2.1-2.3 \mathrm{~V} / \mathrm{m}$ with the number of high-intensity spots less than 30 because of the narrow width.

For all the three defects, the larger the defect in the TATB energetic material is, the stronger the modulation to incident laser is, which is easier to lead to the generation of hot spots. Furthermore, spherical pore defects when $r=1.8 \mu \mathrm{m}$ with 


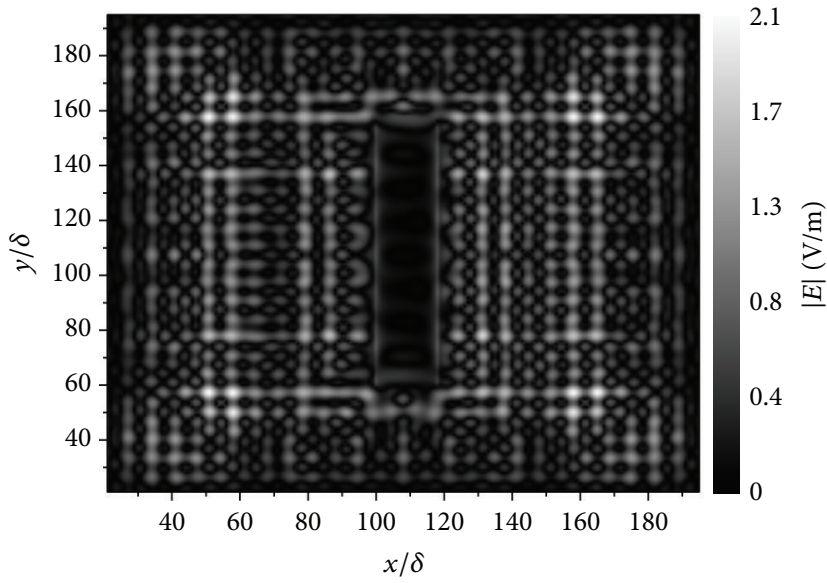

(a)

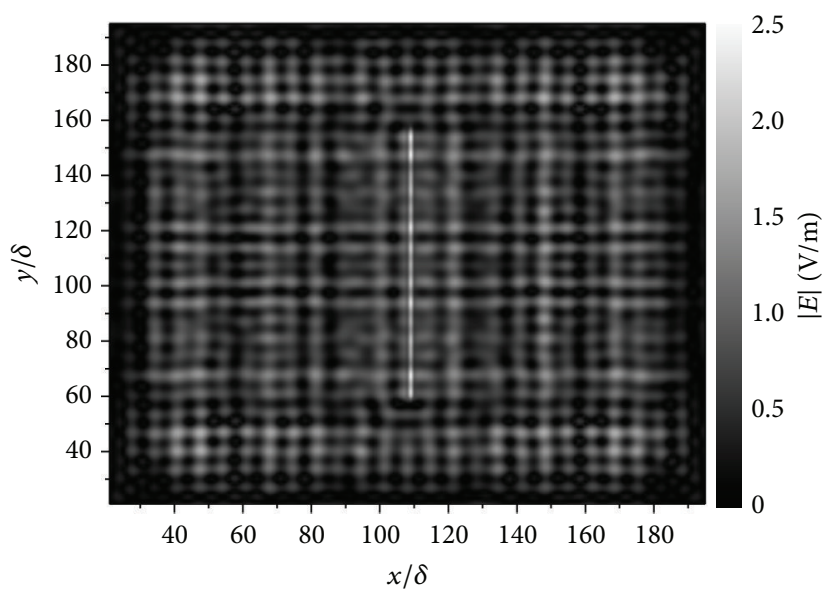

(c)

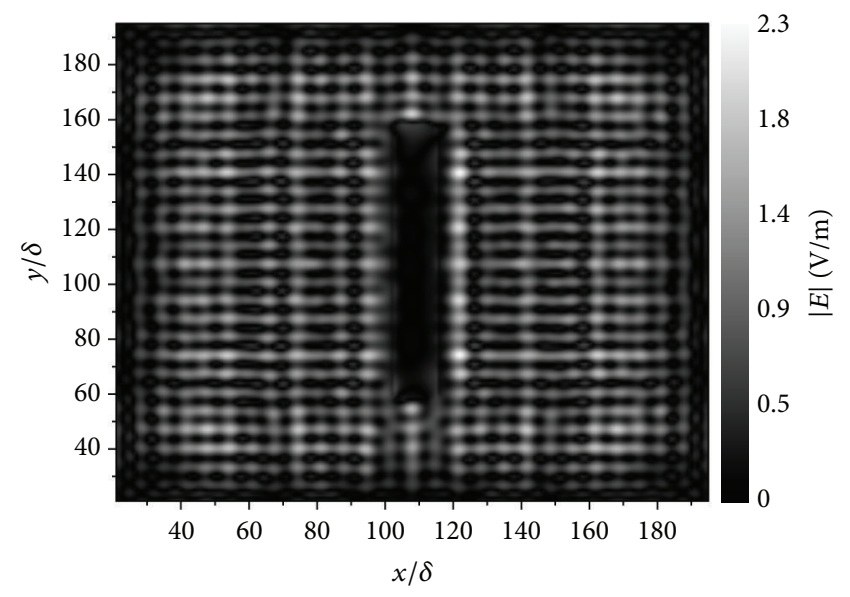

(b)

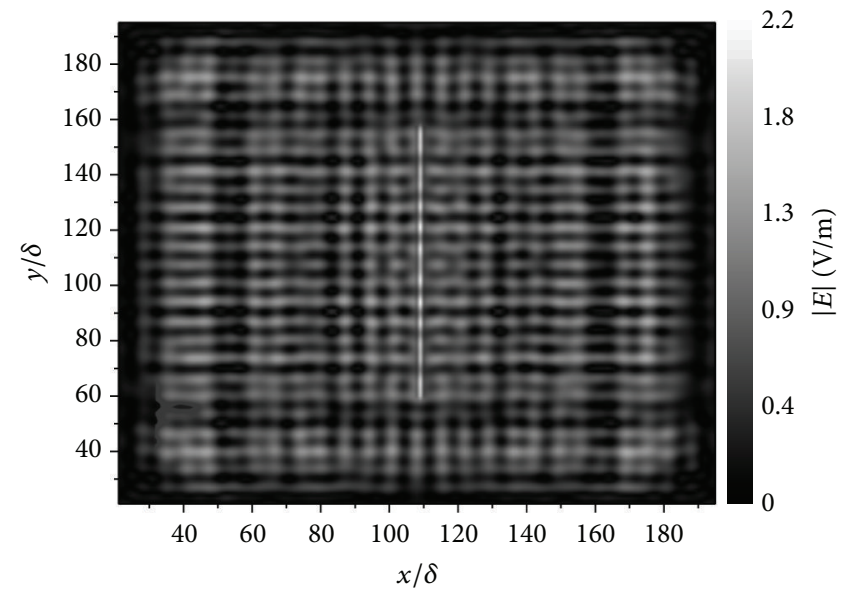

(d)

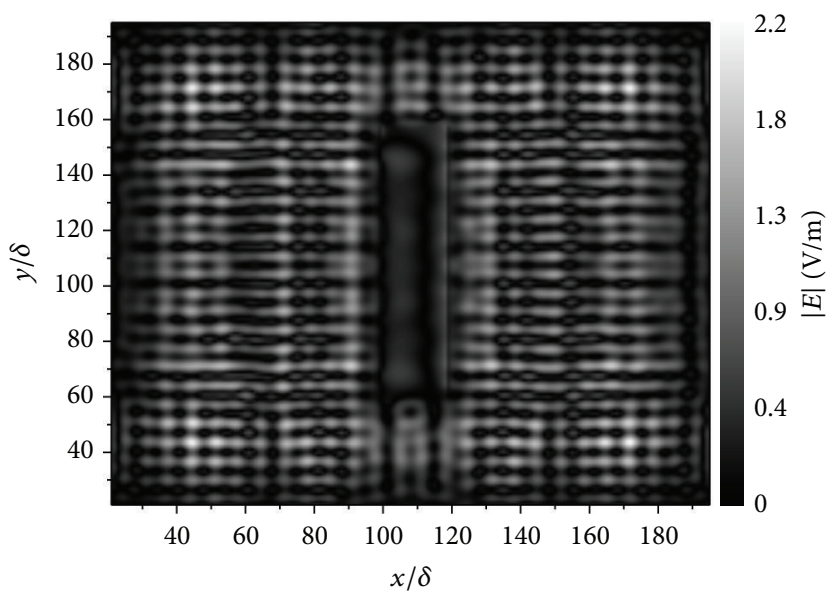

(e)

FIGURE 8: Electric field amplitude distributions in the $z$ plane of $|E|_{\max }$ of cracks with different Rck at the length $l=100 \delta$ and width $w=20 \delta$ : (a) Rck =1, Plane $z 200$; (b) Rck = 2, Plane $z 187$; (c) Rck = 3, Plane $z 141$; (d) Rck = 4, Plane $z 121$; (e) Rck = 5, Plane $z 193$. 
depth/radius ratio from 0.75 to 1.75 and crater defects when $a=3 \mu \mathrm{m}$ with depth/width ratio from 0.5 to 1.0 are easier ways to generate hot spots than crack defects with $w=l / 5$.

\section{Conflict of Interests}

The authors declare that there is no conflict of interests regarding the publication of this paper.

\section{Acknowledgments}

This study was financially supported by the National Natural Science Foundation of China (Grant no. 11372289) and the NSAF Joint Foundation of China (Grant no. U1330108).

\section{References}

[1] A. A. Brish, I. A. Galeev, B. N. Zaitsev, E. A. Sbitnev, and L. V. Tatarintsev, "Mechanism of initiation of condensed explosives by laser radiation," Combustion, Explosion, and Shock Waves, vol. 5, no. 4, pp. 326-328, 1972.

[2] L. C. Yang and V. J. Menichelli, "Detonation of insensitive high explosives by a q-switched ruby laser," Applied Physics Letters, vol. 19, no. 11, pp. 473-475, 1971.

[3] R. J. Harrach, "Estimates on the ignition of high-explosives by laser pulses," Journal of Applied Physics, vol. 47, no. 6, pp. 24732482, 1976.

[4] M. W. Chen, S. You, K. S. Suslick, and D. D. Dlott, "Hot spot generation in energetic materials created by long-wavelength infrared radiation," Applied Physics Letters, vol. 104, no. 6, Article ID 061907, 2014.

[5] C.-L. Zhang, X.-D. Yuan, X. Xiang et al., "Rear-surface light intensification caused by a Hertzian-conical crack in 355-nm silica optics," Chinese Physics B, vol. 21, no. 9, Article ID 094213, 2012.

[6] P. Kosmas, Y. Wang, and C. Rappaport, "Three-dimensional FDTD model for GPR detection of objects buried in realistic dispersive soil," in Detection and Remediation Technologies for Mines and Minelike Targets IV, vol. 4742 of Proceedings of SPIE, pp. 330-338, April 2002.

[7] L. Li, X. Xiang, X. Zu, X. Jiang, X. Yuan, and W. Zheng, "The electric field modulation by three-dimensional crack on fused silica subsurface," Optik, vol. 122, no. 16, pp. 1423-1425, 2011.

[8] J.-R. Hua, L. Li, X. Xiang, and X.-T. Zu, "Three-dimensional numerical simulation of light field modulation in the vicinity of inclusions in silica subsurface," Wuli Xuebao/Acta Physica Sinica, vol. 60, no. 4, Article ID 044206, 2011.

[9] K. Yee, "Numerical solution of initial boundary value problems involving Maxwell's equations in isotropic media," IEEE Transactions on Antennas and Propagation, vol. 14, no. 3, pp. 302-307, 1966.

[10] J. W. Pyper, H. M. Buettner, C. J. Cerjan, J. S. Hallam, and R. J. King, "The measurement of bound and free moisture in organic materials by microwave methods," in Proceedings of the International Symposium on Moisture and Humidity, Washington, DC, USA, 1985.

[11] J.-P. Berenger, "A perfectly matched layer for the absorption of electromagnetic waves," Journal of Computational Physics, vol. 114, no. 2, pp. 185-200, 1994. 

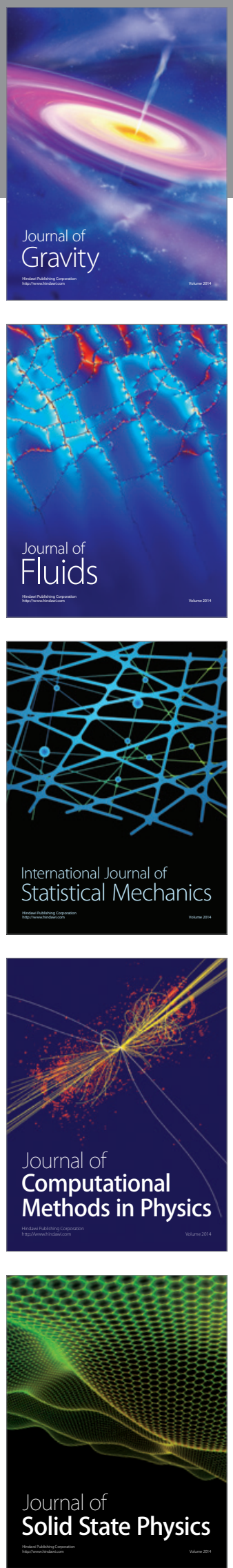

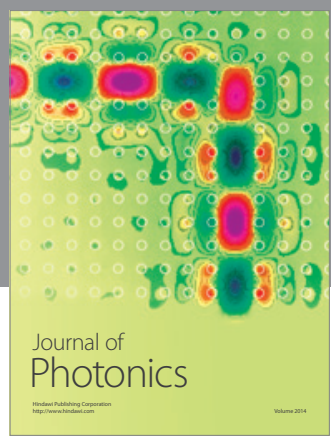

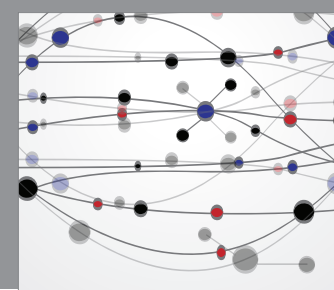

The Scientific World Journal

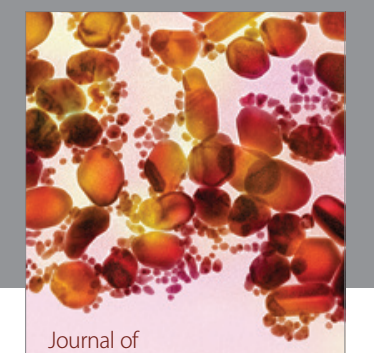

Soft Matter
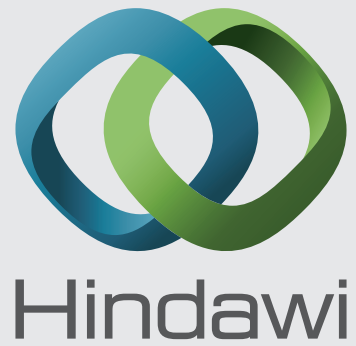

Submit your manuscripts at

http://www.hindawi.com
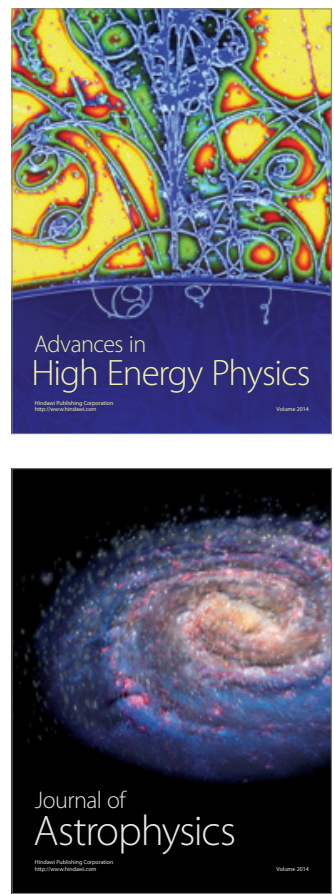
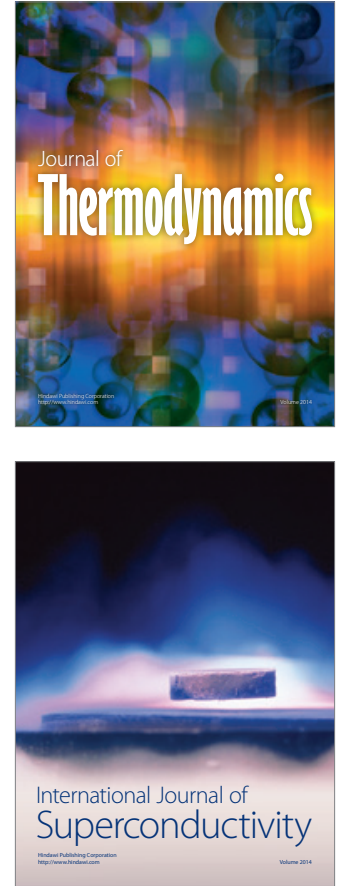
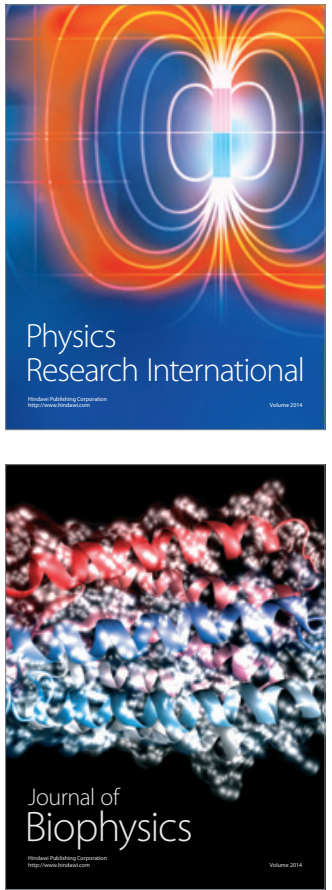
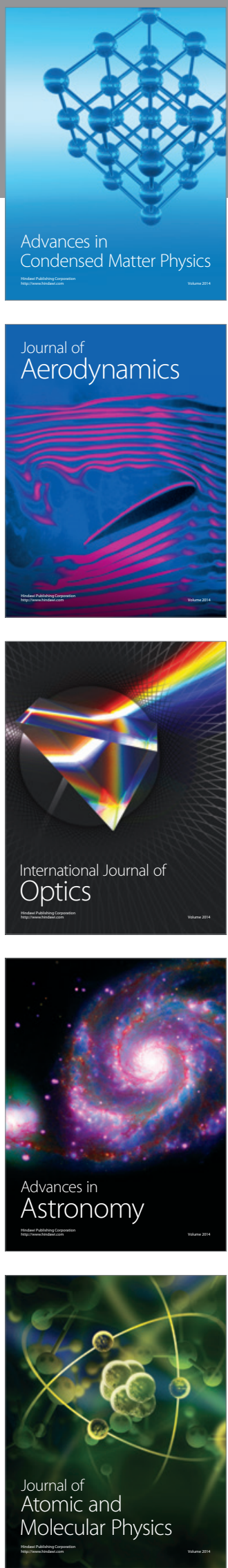